\title{
Sepsis und toxisches Schocksyndrom bei Kindern und Jugendlichen
}

\author{
Markus Hufnagel
}

\section{Definition}

Nach der neuen Sepsis-3-Definition aus dem Jahr 2016 ist die Sepsis eine lebensbedrohliche Organdysfunktion, welche durch eine unkontrollierte Reaktion des Körpers (früher auch als systemische entzündliche Reaktion des Organismus oder SIRS, systemic inflammatory response syndrome bezeichnet) auf eine Infektion mit Bakterien, Viren, Pilzen oder Parasiten hervorgerufen wird. Bei der Sepsis ist die Reaktion des Organismus so unkontrolliert, dass es zu Temperaturerhöhungen oder Untertemperatur, Tachykardie/Bradykardie, Tachypnoe/Beatmungsnotwendigkeit, arterieller Hypotension, disseminierter intravasaler Gerinnung und Multiorganversagen kommen kann.

Sepsis Die Übertragung der neuen Sepsis-3-Definition auf das Kindesalter ist problematisch, da die Definition die Anwendung eines Scores (sogenannter SOFA-Score [sequential organ failure assessment]) voraussetzt, der für das Kindesalter nicht evaluiert ist. Diagnostische Kriterien einer Sepsis im Kindesalter sind von daher unverändert die SIRS-Kriterien nach der International Consensus Conference on Pediatric Sepsis aus dem Jahr 2001 (adaptiert nach der AWMF S2kLeitlinie 024-025 Sepsis bei Kindern jenseits der Neonatalperiode, Dezember 2015; Tab. 1):

1. Hyperthermie $\left(>38,5{ }^{\circ} \mathrm{C}\right)$ oder Hypothermie $\left(<36,0{ }^{\circ} \mathrm{C}\right)$

2. Tachykardie (Herzfrequenz $>2 \mathrm{SD}$ über der altersentsprechenden Norm, Tab. 1) oder Bradykardie (Herzfrequenz $<10$. Perzentile) ohne andere Erklärung im Alter $<1 \mathrm{Jahr}$

3. Tachypnoe (Atemfrequenz $>2$ SD über der altersentsprechenden Norm, Tab. 1)
M. Hufnagel ( $₫)$

Sektion für Pädiatrische Infektiologie und Rheumatologie,

Universitätsklinikum Freiburg, Zentrum für Kinder- und

Jugendmedizin, Freiburg, Deutschland

E-Mail: markus.hufnagel@uniklinik-freiburg.de
4. Leukozytose oder Leukozytopenie (nicht bei chemotherapiebedingter Leukozytopenie) oder Linksverschiebung ( $>10 \%$ unreife Neutrophile),

5. Nachweis oder Verdacht auf eine Infektion

Im Kindesalter werden mindestens 2 der Kriterien 1-4 gefordert, wobei eines der Kriterien die abnormale Körpertemperatur oder die pathologische Leukozytenzahl sein muss.

Neben den SIRS-Kriterien als Folge einer Infektion muss nach der neuen Sepsis-3-Definition zusätzlich eine Organdysfunktion vorliegen. Organdysfunktionen können betreffen:

1. Kardiovaskuläre Organdysfunktion (siehe Definition des septischen Schocks)

2. Respiratorische Dysfunktion:

a. $\mathrm{PaO}_{2} / \mathrm{FiO}_{2}<300$ in Abwesenheit eines zyanotischen Herzfehlers oder einer präexistierenden Lungenerkrankung oder

b. Notwendigkeit einer nichtelektiven invasiven oder nichtinvasiven mechanischen Beatmung

\section{Neurologische Dysfunktion:}

a. Glasgow Coma Scale (GCS) $\leq 11$ oder akute Vigilanzstörung (mit Abnahme des GCS $>3$ Punkte gegenüber dem Ausgangswert) oder

b. Krampfanfall

\section{Hämatologische Dysfunktion:}

a. Thrombozytopenie $<100.000 / \mu 1$ oder Abnahme um $>50 \%$ gegenüber dem höchsten Wert in den vorangegangenen 3 Tagen oder

b. International Normalized Ratio (INR) $>2$

5. Renale Dysfunktion: Serum-Kreatinin $\geq 2$-fache der altersentsprechenden Norm oder mindestens 2-facher Anstieg gegenüber dem Ausgangswert

6. Hepatische Dysfunktion: Gesamt-Bilirubin $\geq 4 \mathrm{mg} / \mathrm{dl}$ (gilt nicht für Neugeborene bis zum 28. Lebenstag) oder 
Tab. 1 Altersabhängige sepsisdefinierende Grenzwerte (5. bzw. 95. Perzentile) für Herzfrequenz, Atemfrequenz, Leukozytenzahlen und systolischen Blutdruck

\begin{tabular}{|c|c|c|c|c|c|}
\hline \multirow[b]{2}{*}{ Altersgruppe } & \multicolumn{2}{|c|}{ Herzfrequenz/min } & \multirow{2}{*}{$\begin{array}{l}\text { Atemfrequenz/min } \\
\text { Tachypnoe }\end{array}$} & \multirow{2}{*}{$\begin{array}{l}\text { Leukozyten-zahl } \\
\left(\times 10^{3} / \mu \mathrm{l}\right)\end{array}$} & \multirow{2}{*}{$\begin{array}{l}\mathrm{RR}_{\text {syst }} \\
(\mathrm{mmHg})\end{array}$} \\
\hline & Tachykardie & Bradykardie & & & \\
\hline $0-6$ Tage & $>180$ & $<100$ & $>50$ & $>34$ & $<65$ \\
\hline 7-28 Tage & $>180$ & $<100$ & $>40$ & $>19,5$ oder $<5$ & $<75$ \\
\hline 1 Monat bis $<1 \mathrm{Jahr}$ & $>180$ & $<90$ & $>50$ & $>17,5$ oder $<5$ & $<65$ \\
\hline $1-<6$ Jahre & $>140$ & n.a. & $>40$ & $>15,5$ oder $<6$ & $<75$ \\
\hline $6-<12$ Jahre & $>130$ & n.a. & $>20$ & $>13,5$ oder $<4,5$ & $<83$ \\
\hline $12-<18$ Jahre & $>110$ & n.a. & $>20$ & $>11$ oder $<4,5$ & $<90$ \\
\hline
\end{tabular}

$R R_{\text {syst }}$ systolischer Blutdruck nach Riva-Rocci; Monat Lebensmonat, Jahr Lebensjahr, n.a. nicht anwendbar

Serum-GPT bzw. ALT $\geq 2$-fache der altersentsprechenden Norm.

Schwere Sepsis Die schwere Sepsis ist nach der neuen Sepsis-3-Definition nicht mehr separat definiert, da nach der neuen Definition bei jeder Sepsis eine gestörte Organdysfunktion vorliegt.

Septischer Schock Beim septischen Schock liegen vor:

1. Schwere kardiovaskuläre Dysfunktion: trotz Gabe von isotoner intravaskulärer Flüssigkeit von $\geq 40 \mathrm{ml} / \mathrm{kg}$ in 1 Stunde

a. Arterielle Hypotonie (systolischer Blutdruck $<5$. Perzentile oder $<2$ SD der altersentsprechenden Norm (Tab. 1) oder

b. Notwendigkeit einer vasoaktiven Therapie, um den systolischen Blutdruck in der Altersnorm zu halten oder

2. Zelluläre/metabolische Dysfunktion mit: mindestens 2 der folgenden klinischen oder laborchemischen Zeichen:

- Metabolische Azidose (Basendefizit $>5 \mathrm{mmol} / \mathrm{l}$ )

- Laktaterhöhung (arteriell gemessen $>2$ SD der Altersnorm)

- Oligurie (Urinausscheidung $<0,5 \mathrm{ml} / \mathrm{kg} / \mathrm{h}$ )

- Verlängerte kapillare Füllungszeit $(>5 \mathrm{~s}$ )

- Diskrepanz von Körperkern- zu peripherer Temperatur $\left(>3{ }^{\circ} \mathrm{C}\right)$

Toxisches Schocksyndrom Das toxische Schocksyndrom ist eine durch bakterielle Exotoxine ausgelöste immunvermittelte, fulminant verlaufende Multisystemerkrankung bei vorher gesunden Patienten. Im Gegensatz zu dem durch Staphylokokken ausgelösten toxischen Schocksyndrom, bei dem die Blutkultur regelhaft steril bleibt, lassen sich bei dem Streptokokken-assoziierten toxischen Schocksyndrom die Bakterien meist im Blutstrom nachweisen.

Der Begriff toxisches Schocksyndrom wurde erstmals 1978 verwendet. Die Krankheit ist aber schon länger bekannt, seit 1929 wurde dafür der Begriff staphylococcal scarlet fever benutzt. Im Jahr 1990 stellte das Centers for
Disease Control and Prevention (CDC) in den USA Diagnosekriterien auf. Die Diagnose ist wahrscheinlich, wenn 5 Kriterien zutreffen, und sie ist gesichert, wenn alle 6 Befunde vorliegen.

Diagnosekriterien für das Staphylokokken-bedingte toxische Schocksyndrom (TSS; modifiziert nach Wharton et al. 1990)

1. Fieber $>38,9{ }^{\circ} \mathrm{C}$

2. Exanthem: diffuse Erythrodermie

3. Hautdesquamation: 1-2 Wochen nach Erkrankungsbeginn, vor allem im Bereich der Handinnenflächen und Fußsohlen

4. Arterielle Hypotension: $<5$. Perzentile der altersentsprechenden Norm

5. Multisystemerkrankung mit Beteiligung von 3 oder mehr der folgenden Organe:

a. Gastrointestinaltrakt: Erbrechen oder Durchfall

b. Muskulatur: Myalgie oder Erhöhung der Kreatinkinase $(>2$ SD der altersentsprechenden Norm)

c. Schleimhäute: vaginale, oropharyngeale oder konjunktivale Hyperämie

d. Niere: Pyurie ( $>5$ Leukozyten $/ \mu$ l) oder Erhöhung des Kreatinins bzw. Harnstoffs ( $>2$ SD der altersentsprechenden Norm)

e. Leber: Bilirubin- oder Transaminasenanstieg ( $>2$ SD der altersentsprechenden Norm)

f. Blut: Thrombozyten $<100.000 / \mu l$

g. ZNS: Bewusstseinsstörung oder Desorientierung

6. Negative bakterielle Diagnostik

a. Negative Blut-, Liquorkultur und negativer Rachenabstrich

b. Negative Serologie für Masern, Rocky-Mountain-Fleckfieber, Leptospirose

Das STSS wurde 1987 erstmalig beschrieben. 1995 stellte das Centers for Disease Control and Prevention (CDC) Diagnosekriterien auf: Bei $1 \mathrm{a}+2 \mathrm{a}+2 \mathrm{~b}$ gilt die Diagnose als sicher, bei $1 b+2 a+2 b$ ist sie wahrscheinlich. 
Diagnosekriterien für das Streptokokken-bedingte toxische Schocksyndrom (STSS; modifiziert nach der Working Group on Severe Streptococcal Infections 1993)

1. Isolierung von Streptokokken der Gruppe A

a. Aus Materialien von normalerweise sterilen Entnahmeorten (Blut, Liquor, Pleura, Aszites, Urin, Synovia, Gewebeprobe)

b. Aus unsterilen Patientenmaterialien (Rachen, Sputum, Vaginalsekret, Hautabstrich)

2. Klinische Kriterien

a. Arterielle Hypotension $(<5$. Perzentile der altersentsprechenden Norm)

b. Mindestens 2 der folgenden Befunde:

- Generalisiertes Exanthem oder Desquamation

- Nekrotisierende Fasziitis oder Gangrän oder Myositis

- ARDS

- Nierenversagen: Kreatininanstieg $\geq 177 \mu \mathrm{mol} / 1$ bzw. $\geq 2 \mathrm{mg} / \mathrm{dl}$ oder $\geq 2 \mathrm{SD}$ der Altersnorm

- Koagulopathie: Thrombozyten $\leq 100.000 / \mu 1$ oder disseminierte intravasale Gerinnung (DIC)

- Leberschädigung: Bilirubin- oder Transaminasenanstieg $\geq 2$ SD der Altersnorm

\section{Epidemiologie}

Die Sepsis bei Kindern ist weltweit eine der häufigsten Todesursachen und zeigt einen Altersgipfel in der Neugeborenen-Periode (betroffen sind vor allem Frühgeborene) und einen im frühen Kindesalter. Die Fallzahl der Sepsis ist in Deutschland in den Jahren 2007-2013 jährlich um 5,7 \% gestiegen. Die adjustierte Krankenhausrate lag bei Neugeborenen bei 1556/100.000, bei den 10- bis 14-Jährigen bei 30/100.000, die Raten beim septischen Schock bei 16,7 bzw. 1,1/100.000. Die Krankenhausletalität der Sepsis betrug im Beobachtungszeitraum bei Neugeborenen 3,9\%, bei 10 bis 14-Jährigen 3,6 \%. Die Krankenhausletalität des septischen Schocks war mit 39,5 \% bzw. 25,0 \% in beiden Altersgruppen deutlich höher. Die Sterblichkeitsrate der Sepsis in Deutschland ist im Zeitraum 2007-2013 für Kinder und Erwachsene um 2,7 \% gesunken. Bis zu 50 \% der Kinder mit Sepsis haben eine chronische Grunderkrankung. Die Zunahme der Sepsis-Fallzahlen ist vor allem auf die steigende Anzahl von immunsupprimierten Patienten zurückzuführen. Auf Kinderintensivstationen wird ein Viertel der Patienten wegen einer Sepsis behandelt, in $20 \%$ handelt es sich dabei um nosokomiale Infektionen. Neben den in Tab. 2 aufgeführten Risikofaktoren spielen für die Entwicklung der Sepsis noch Unterernährung, chronische Krankheiten, Traumen, Verbrennungen, vorausgegangene Virusinfektionen und lang andauernde Krankenhausaufenthalte eine Rolle.
Auch beim Erregerspektrum ist ein Wandel zu verzeichnen. Gramnegative Erreger (25\% der kindlichen SepsisFälle; vor allem Rückgang an Haemophilus influenzae Typ b seit Einführung der HiB-Impfung in die Grundimmunisierung des Säuglings) verlieren an Bedeutung, während grampositive Erreger (65\%, am häufigsten koagulasenegative Staphylokokken, aber auch Enterokokken) und Pilze (10\%) zunehmen. Unter den gramnegativen Enterobacteriaceae ist in den letzten Jahren eine Zunahme der ESBL-Bildner (extended-spectrum beta-Laktamase) zu verzeichnen. Werden Erwachsene eingeschlossen, so ist die Sepsis für mehr Todesfälle verantwortlich als alle Krebserkrankungen und fordert genauso viele Todesfälle wie die koronare Herzkrankheit.

Das TSS betrifft fast ausschließlich (95\%) junge Frauen, $90 \%$ sind zwischen 15 und 20 Jahre alt. Bis 1985 hatten $80 \%$ der betroffenen Frauen zum Zeitpunkt der Erkrankung ihre Menses, $90 \%$ der Betroffenen benutzten Tampons. Diese starke Assoziation mit dem Risikofaktor Tampon fand in dem Begriff menstruelles TSS ihren Niederschlag. Die Inzidenz in den USA wurde mit 6,2-12,3:100.000 Frauen im Alter von 15-44 Jahren angegeben. Mit Aufklärung und sinkendem Tampon-Verbrauch und der Einführung geringer absorbierender Tampons sanken ab Mitte der 1980er-Jahre die Inzidenzzahlen. Seit dem Jahr 2000 wird in den USA wieder eine Zunahme der Inzidenz beobachtet (3,4:100.000 im Jahr 2003). Gleichzeitig fällt eine Zunahme des nichtmenstruellen TSS auf. Andere Risikofaktoren, wie vorangegangene Haut- oder Weichteilinfektionen, Verbrennungen, Infektionskrankheiten des Respirationstrakts, Zustand nach Geburt, Abort oder nach operativem Eingriff spielen ebenfalls eine Rolle. Das Verhältnis von nichtmenstruellem zu menstruellem TSS beträgt heutzutage 60:40\%.

Die Inzidenz der invasiven Streptokokken-Infektionen in Nordamerika, Skandinavien und Großbritannien nimmt seit Ende der 1980er-Jahre zu, aus Ontario ist eine Inzidenz von 0,09-0,5:100.000 Einwohner publiziert. Epidemiologische Zahlen für Deutschland liegen nicht vor. Im Rahmen einer europäischen Studie (Strep-EURO) zu schweren AStreptokokken-Infektionen wurde das STSS in $13 \%$ dieser Fälle beobachtet. Das STSS ist im Kindesalter selten, betroffen sind in erster Linie Erwachsene im Alter von 20-50 Jahren. Im United Kingdom waren über einen 13-Monatszeitraum in den Jahren 2008-2009 etwa gleich viele TSS und STSS-Fälle prospektiv gesammelt worden. Kinder mit STSS waren jünger (durchschnittliches Alter 3,8 Jahre vs. 9,5 Jahre). $28 \%$ der STSS-Fälle verliefen tödlich, während keiner der TSS-Patienten verstarb. Virusinfektionen, z. B. Windpocken und Verbrennungen sind Risikofaktoren für ein STSS. In der Hälfte der STSS-Fälle bestehen potenzielle Eintrittspforten für Streptokokken, z. B. (Operations-) Wunden, Uterusschleimhaut nach Geburt oder Tonsillopharyngitis. Ein vermuteter Zusammenhang zwischen STSS und 
der Einnahme von nichtsteroidalen Antiphlogistika konnte in 2 epidemiologischen Studien aus den USA und Skandinavien nicht bestätigt werden.

\section{Ätiologie}

Das Erregerspektrum der Sepsis ist abhängig vom Alter. Bei der Sepsis des Neugeborenen findet man andere Keime (vor allem B-Streptokokken und Escherichia coli) als bei der Sepsis des Säuglings und des Kleinkindes (vor allem Pneumokokken, Haemophilus influenzae und Meningokokken ohne entsprechende Impfungen - , sonst Staphylokokken, Streptokokken und Salmonellen). In Mitteleuropa sind bei gesunden Kindern die häufigsten Sepsis-Erreger Pneumokokken, Streptokokken, Meningokokken, Staphylokokken, Haemophilus influenzae und Salmonella spp. Tropenspezifische Sepsis-Erreger sind Salmonella typhi, Yersinia pestis, Burkholderia pseudomallei und Malaria.

Bei Vorliegen einer Grundkrankheit wandelt sich das Erregerspektrum. In Tab. 2 sind einige Krankheiten und Situationen aufgeführt, bei denen das Immunsystem endogen und exogen beeinträchtigt ist und bei denen es leichter zu einer Sepsis kommen kann. Polymikrobielle Septikämien treten bei Hochrisiko-Patienten (Malignom, Neutropenie, Erkrankungen des Gastrointestinaltraktes, zentrale Venenkatheter) auf.

Das klassische toxische Schocksyndrom (toxic shock syndrome, TSS) wird durch Exotoxine von Staphylococcus aureus, in Ausnahmefällen auch von koagulasenegativen Staphylokokken oder Yersinia pseudotuberculosis ausgelöst. Das streptokokken-bedingte toxische Schocksyndrom (streptococcal toxic shock syndrome, STSS) wird von Streptokokken, überwiegend der Gruppe A, aber auch der Gruppen B, C, F, G und Streptococcus sanguis, ausgelöst. Ein synonym verwendeter Begriff ist toxic shock-like syndrome (TSLS).

\section{Pathogenese}

Die Pathogenese der Sepsis hängt zum einen von der Virulenz des Mikroorganismus und von Wirtsfaktoren ab. Zu den Wirtsfaktoren gehören der Immunstatus und die Anwesenheit weiterer Risikofaktoren, wie Grunderkrankungen, aber auch Lebensalter, Geschlecht und genetischer Hintergrund.

Die Pathogenese der Sepsis ist am besten bei gramnegativen und grampositiven Bakterien untersucht und stellt ein Modell dar (Abb. 1). Trotz Gemeinsamkeiten ist die Pathogenese der grampositiven Sepsis signifikant unterschiedlich zur gramnegativen Sepsis. Die Unterschiede betreffen in erster Linie die Initiierung der Sepsis-Kaskade und die unterschiedlichen Strategien der Bakterien, die Immunantwort des Wirts zu umgehen. So können z. B. Exotoxine von grampositiven Erregern als Superantigene agieren und die Freisetzung proinflammatorischer Mediatoren durch T-Lymphozyten und Monozyten/Makrophagen auslösen und so ein toxisches Schocksyndrom verursachen. Nichtmethylierte
Tab. 2 Erregerspektrum der Sepsis bei Risikofaktoren

\begin{tabular}{|c|c|}
\hline Risikofaktoren & Erreger \\
\hline Neutropenie & $\begin{array}{l}\text { Escherichia coli, Klebsiella spp., } \\
\text { Pseudomonas aeruginosa, Acinetobacter spp., } \\
\text { Enterobacter spp., Serratia marcescens, } \\
\text { Aeromonas hydrophila, Staphylococcus } \\
\text { aureus, Staphylococcus epidermidis, } \\
\text { Corynebacterium spp., } \alpha \text {-hämolytische } \\
\text { Streptokokken, Candida spp., Aspergillus } \\
\text { spp., Trichosporon spp., Fusarium, Alternaria, } \\
\text { Pseudoallescheria }\end{array}$ \\
\hline Phagozytendefekte & $\begin{array}{l}\text { Staphylococcus aureus, Streptococcus spp., } \\
\text { Serratia marcescens, Escherichia coli, } \\
\text { Burkholderia cepacia, Salmonella spp. }\end{array}$ \\
\hline T-Zell-Defekte & $\begin{array}{l}\text { Listeria monocytogenes, Mycobacterium } \\
\text { tuberculosis, nichttuberkulöse } \\
\text { Mykobakterien, Salmonella spp., } \\
\text { Cryptococcus neoformans, Histoplasma } \\
\text { capsulatum, Coccioides immitis, } \\
\text { Cytomegalie-Virus, Herpes-simplex-Virus, } \\
\text { Strongyloides stercoralis }\end{array}$ \\
\hline Komplementdefekte & $\begin{array}{l}\text { Streptococcus pneumoniae, Haemophilus } \\
\text { influenzae, Neisseria meningitidis, Neisseria } \\
\text { gonorrhoeae }\end{array}$ \\
\hline Milzdefekt & $\begin{array}{l}\text { Streptococcus pneumoniae, Haemophilus } \\
\text { influenzae, Neisseria meningitidis }\end{array}$ \\
\hline Venenkatheter & $\begin{array}{l}\text { Staphylococcus aureus, Staphylococcus } \\
\text { epidermidis, Corynebacterium spp., Candida } \\
\text { spp. }\end{array}$ \\
\hline Blasenkatheter & $\begin{array}{l}\text { Escherichia coli, Enterokokken, } \\
\text { Streptococcus saprophyticus }\end{array}$ \\
\hline Peritonealkatheter & $\begin{array}{l}\text { Staphylococcus aureus, Staphylococcus } \\
\text { epidermidis, Pseudomonas aeruginosa }\end{array}$ \\
\hline Verbrennungen & $\begin{array}{l}\text { Pseudomonas aeruginosa, Staphylococcus } \\
\text { epidermidis, Candida spp. }\end{array}$ \\
\hline Hautinfektion & $\begin{array}{l}\text { Streptococcus pyogenes, Staphylococcus } \\
\text { aureus, Pseudomonas aeruginosa }\end{array}$ \\
\hline $\begin{array}{l}\text { Abdomineller } \\
\text { Fokus }\end{array}$ & Enterobacteriaceae, Anaerobier \\
\hline i.v.-Drogenabusus & $\begin{array}{l}\text { Staphylococcus aureus, Streptococcus spp., } \\
\text { Pseudomonas aeruginosa }\end{array}$ \\
\hline
\end{tabular}

CpG-Abschnitte der bakteriellen DNA induzieren ebenfalls direkt eine Sekretion proinflammatorischer Mediatoren, außerdem können B-Zellen nicht nur durch nichtmethylierte CpG-Abschnitte, sondern auch durch Peptidoglykane von grampositiven Bakterien direkt stimuliert werden. Für gramnegative Bakterien sind vergleichbare Mechanismen bisher nicht bekannt.

Nach einer Kolonisation von Schleimhäuten oder Fremdkörpern (z. B. zentrale Venenkatheter) folgt die Gewebeinvasion, die zu einer Bakteriämie führt. Verschiedene Bestandteile der Bakterienzellmembran, die bei der Abwehr durch das Immunsystem freigesetzt werden, wirken als Auslöser der Sepsis-Kaskade. Bei gramnegativen Erregern sind dies Lipopolysaccharide (LPS oder Endotoxin) und Lipoproteine. Ein LPS-Äquivalent konnte bisher bei grampositiven Erregern nicht identifiziert werden, die Bedeutung von Lipotei- 


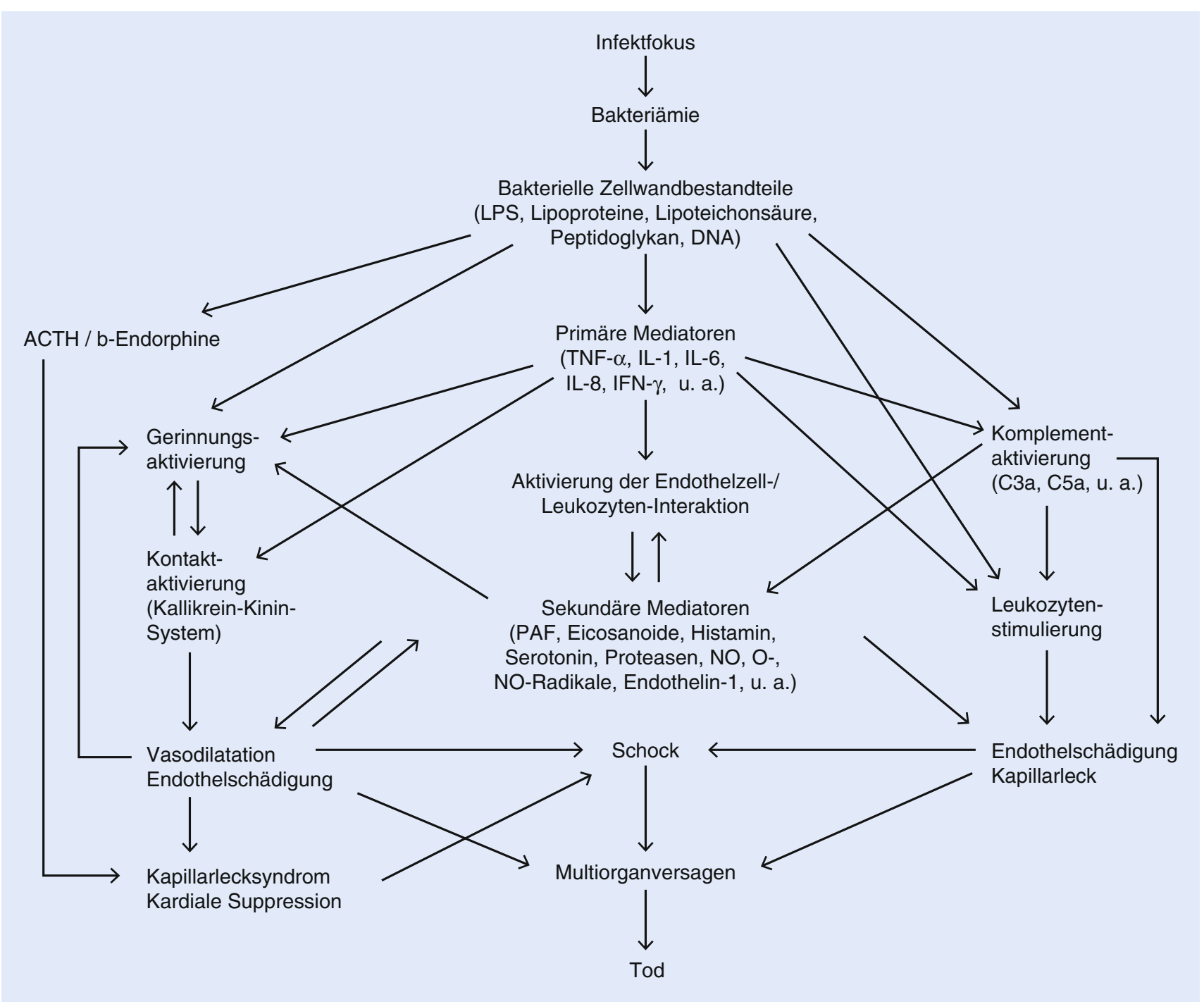

Abb. 1 Pathogenese-Modell der bakteriellen Sepsis. (Modifiziert nach Sáez-Llorens und McCracken 1993)

chonsäure und Peptidoglykan wird kontrovers beurteilt. Am Beispiel von LPS können die weiteren Reaktionsschritte erläutert werden:

Das LPS bindet an ein in der Leber gebildetes Serumprotein, das LPS-bindende Protein (LBP). Der LPS-LBP-Komplex bindet über das Glykoprotein CD14 und MD2 an Zielzellen, in erster Linie Monozyten/Makrophagen, aber auch an Endothelzellen, polymorphkernige neutrophile Granulozyten (PMN) und andere humane Zellen. Als essenzielle Ko-Rezeptoren auf Monozyten/Makrophagen fungieren sog. Toll-like-Rezeptoren (TLR). Sie gehören zu den PRR (pattern-recognition receptors), erkennen PAMPs (pathogenassociated molecular patterns, d. h. exogene Liganden auf Mikroorganismen) oder auch DAMPs (danger-associated molecular patterns, d. h. endogene Liganden der Wirtszellen) und spielen die zentrale Rolle bei der Vermittlung der Immunantwort im Rahmen der Sepsis. Daneben spielen wei- tere PRR-Moleküle der unspezifischen Immunantwort, z. B. „C-type lectin“-Rezeptoren (CLR), intrazelluläre ,nucleotide-binding oligomerization domain-like“-Rezeptoren (NLR), „retinoic acid-inducible gene-like“-Rezeptoren (RLR) und das alternative Komplementsystem eine Rolle in der Initiierung der Sepsis-Kaskade. Aktivierte Monozyten/Makrophagen, aber auch Endothelzellen, Leukozyten und Thrombozyten sezernieren primäre endogene Mediatoren. Die wichtigsten Mediatoren in der frühen, als hyperreaktive Immunantwort bezeichneten Phase sind proinflammatorische Zytokine, vor allem Tumornekrosefaktor(TNF)- $\alpha$, Interleukin(IL)-1 $\beta$, IL-2, IL-6, IL-8, IL-12, IL-17, IL-18, Interferon(IFN)- $\gamma$, macrophage migration inhibitory factor (MIF) und High-mobility-group-box-1-Protein (HMGB-1). Die endogenen Mediatoren sind direkt verantwortlich für eine Vielzahl von Symptomen wie Fieber, myokardiale Suppression, Kapillarleck, Gerinnungsaktivierung und Erhöhung 
der Akute-Phase-Proteine. Über eine vermehrte Expression von Adhäsionsmolekülen (Selektine, Integrine) auf Endothelzellen und Granulozyten wird die Adhärenz von Monozyten und Granulozyten an Endothelzellen gesteigert. Dies bewirkt konsekutiv eine Degranulation und Freisetzung von Enzymen, Proteasen und Oxidanzien, die zu einer Endothelzellschädigung führt. Die Folge ist eine gesteigerte Gefäßpermeabilität mit Verlust von intravasaler Flüssigkeit in das Interstitium, es entsteht ein sog. Kapillarlecksyndrom (sog. hypovolämischer Schock). Die Stimulierung von Granulozyten wird über aktiviertes Komplement unterstützt. Die Interaktion von Endothelzellen mit Granulozyten oder Thrombozyten bewirkt die Freisetzung einer Reihe sekundärer endogener Mediatoren wie plättchenaktivierender Faktor (PAF), Leukotriene, Prostaglandine (Prostazyklin, Thromboxan), Histamin, Serotonin, Proteasen, freie Sauerstoffund Stickstoffradikale. Vor allem über Stickoxid (NO) wird eine Vasodilatation der Blutgefäße vermittelt, die durch eine Kontaktaktivierung mit vermehrter Bradykinin-Sekretion unterstützt wird. Die Kontaktaktivierung (sog. KallikreinKinin-System) wird über eine Aktivierung des Gerinnungsfaktors XII (Hageman-Faktor) induziert. Faktor XII ist auch das Startermolekül der Aktivierung der intrinsischen Gerinnungskaskade. Hauptursache für die aktivierte Gerinnung ist jedoch die Anstoßung der extrinsischen Gerinnungskaskade über den durch Endotoxin aktivierten Tissue factor (TF). Die Aktivierung beider Gerinnungskaskaden führt zum klinischen Bild einer disseminierten intravasalen Gerinnung (disseminated intravasal coagulation, DIC). Die Bildung von Mikrothromben über die aktivierte Gerinnung wird durch den Verbrauch von Inhibitoren der Gerinnung wie Antithrombin III, Protein C, Protein S, C1-Esteraseinhibitor und tissue factor pathway inhibitor (TFPI) unterstützt. Das Auflösen der Mikrothromben verhindert eine Inhibition der Fibrinolyse über eine Stimulierung des PlasminogenAktivator-Inhibitors (PAI-1). Der Verbrauch von Gerinnungsfaktoren und die Thrombozytopenie erklären die vermehrte Blutungsneigung im Rahmen der Sepsis. Das Blutungsrisiko ist bei Neugeborenen und Säuglingen mit Sepsis am höchsten. Zur Thrombozytopenie kommt es durch den Verbrauch der Blutplättchen in den Fibrinablagerungen, durch die Adhäsion an alterierten Endothelzellen und durch eine vermehrte Sequestrierung in Leber und Lunge. Die DIC mit Bildung und Ablagerung von Fibrinthromben in den Organen spielt zusätzlich zur Minderperfusion die wesentliche pathogenetische Rolle beim Multiorganversagen.

Das aktuelle pathogenetische Konzept der Sepsis geht davon aus, dass proinflammatorische Zytokine per se physiologische Reaktionen des Körpers als Ausdruck der frühen Immunantwort auf die Invasion von mikrobiellen Erregern triggern. Eine überschießende und unkontrollierte Produktion/Aktivierung von proinflammatorischen Zytokinen führt zum SIRS. Die systemische Reaktion wiederum setzt in einer späten Phase der Sepsis, die als Phase der hyporeaktiven Immunantwort bezeichnet wird, antiinflammatorische Zytokine/Mediatoren frei. Diese Phase wird auch als compensatory anti-inflammatory response syndrome (CARS) bezeichnet. $\mathrm{Zu}$ den wichtigsten Mediatoren gehören IL-4, IL-10, IL-11, IL-13, transforming growth factor $\beta$ (TGF- $\beta$ ), lösliche TNF-Rezeptoren (sTNFR) und IL-1-Rezeptorantagonisten (IL-1RA), die die proinflammatorische Antwort herunterregeln und die systemische Antwort des Organismus beenden. Die Herunterregulation der proinflammatorischen Immunantwort wird durch neuronale Mediatoren, z. B. Glukokortikoide, Adrenalin, Acetylcholin, VIP (vasoactive intestinal peptide) und PACAP (pituitary adenylate cyclase activating peptide), unterstützt. Ein Überwiegen der antiinflammatorischen Antwort kann zu einer Suppression der Immunantwort führen und die Sepsis-Kaskade unterhalten. Zusätzlich wird eine vermehrte Apoptose von CD4-, und CD-8-positiven T-Lymphozyten, B-Lymphozyten und follikulären dendritischen Zellen induziert, die zu einer Anergie führt und die Immunsuppression unterstützt. Als Folge der Immunsuppression können in der Spätphase der Sepsis sekundäre Infektionen oder Virusreaktivierungen auftreten. Nur eine Balance zwischen proinflammatorischen und antiinflammatorischen Mediatoren führt zur Homöostase des Immunsystems, die die Erregerelimination ohne überschießende und somit schädliche Entzündungsantwort gewährleistet (Abb. 2).

Septischer Schock Der septische Schock ist eine Verteilungsstörung des Blutvolumens mit Zunahme des venösen Blutpools durch Vasodilatation im großen Kreislauf. Die Folge ist eine Störung der Mikrozirkulation durch Öffnen von Shunts mit verminderter Perfusion und verminderter $\mathrm{O}_{2}$-Aufnahme bei gleichzeitig vermehrtem $\mathrm{O}_{2}$-Bedarf (sog. distributiver Schock). Zur Aufrechterhaltung der Gewebeoxygenierung reagiert der Körper über eine vermehrte Katecholaminsekretion mit einer Steigerung des Herzzeitvolumens (sog. hyperdynamer Schock). Diese hyperdyname Reaktion der Makrozirkulation kennzeichnet die frühe oder warme Phase des septischen Schocks. Im Kindesalter erfolgt die Steigerung des Herzzeitvolumens primär über die Herzfrequenz, da die Steigerungskapazität des Schlagvolumens beschränkt ist. Allerdings ist auch dieser Kompensationsmechanismus durch die Abhängigkeit der Perfusion der Koronargefäße in der diastolischen Füllung begrenzt. Kann das Herzzeitvolumen die Verteilungsstörung nicht mehr kompensieren und ist die Myokardfunktion eingeschränkt, entsteht die sog. späte oder kalte Phase des septischen Schocks (auch als hypodynamer Schock bezeichnet), die zu einem Multiorganversagen und evtl. zum Tod führt (Kap. > „Akute Herz-Kreislauf-Insuffizienz und Schock bei Kindern und Jugendlichen"). 


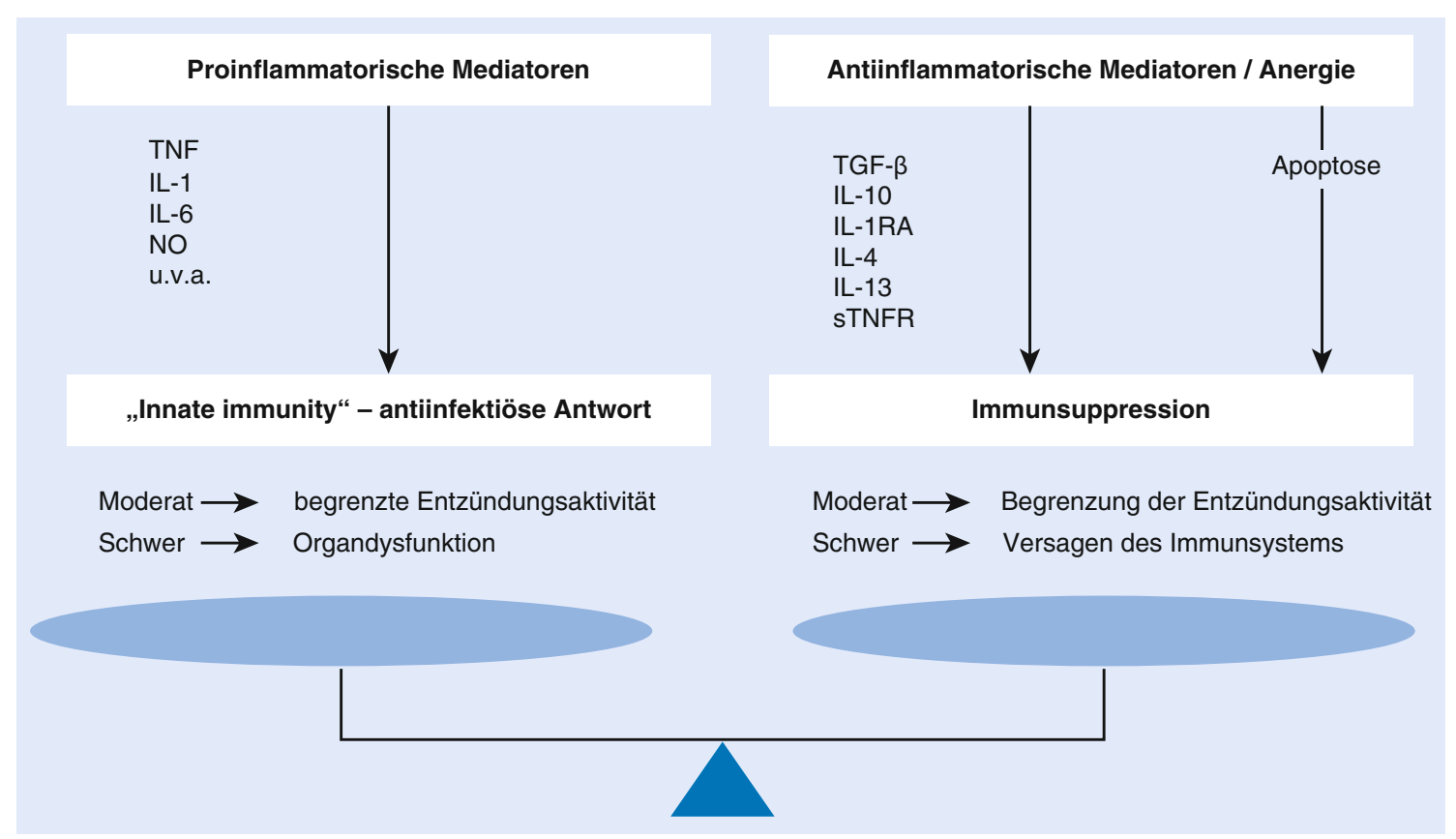

Abb. 2 Gleichgewicht zwischen proinflammatorischer und antiinflammatorischer Immunantwort bei Sepsis. (Modifiziert nach Annane et al. 2005)

Toxisches Schocksyndrom Die toxischen Schocksyndrome werden durch Exotoxin-produzierende Staphylokokken und Streptokokken ausgelöst, die eine überschießende Immunantwort, vermutlich superantigenvermittelt, hervorrufen. Beim TSS werden Staphylokokken-Stämme isoliert, die verschiedene Exotoxine produzieren können. Das wichtigste Toxin ist das TSST-1 (toxic shock syndrome toxin-1). Es wird in $>90 \%$ der menstruellen TSS- und in zwei Drittel der nichtmenstruellen TSS-Fälle isoliert, hingegen produzieren nur $<30 \%$ der nicht TSS auslösenden Staphylokokken das Toxin. Weitere Staphylokokken-Exotoxine, die ein TSS auslösen können, sind die sog. StaphylokokkenEnterotoxine A, B und C (SEA, SEB, SEC). Diese Toxine sind primär verantwortlich für die mit Staphylokokken assoziierten Nahrungsmittelintoxikationen. TSST-1 und SEF werden synonym verwendet und beschreiben das gleiche Toxin. Auslösende Exotoxine des STSS sind streptococcal pyrogenic exotoxins (Spe-A, Spe-B, Spe-C u. a., sowie M-Proteinfragmente).

Die superantigenvermittelte Immunantwort im Rahmen des TSS weist im Gegensatz zu herkömmlichen Antigenen folgende Besonderheiten auf:

- Es muss keine vorherige Aufarbeitung durch antigenpräsentierende Zellen (APC) erfolgen.

- Superantigene induzieren eine unspezifische, polyklonale Aktivierung eines ungleich größeren Anteils (5-20\%) an ruhenden T-Zellen über eine simultane Bindung an MHC Klasse-II-Moleküle und T-Zellrezeptoren.

Drei verschiedene Mechanismen des Exotoxin-vermittelten Schockgeschehens werden unterschieden:

1. Aktivierte T-Zellen und APC setzen vermehrt proinflammatorische Zytokine vom Th1-Typ (vor allem TNF- $\alpha$, TNF- $\beta$, IL-1, IL-2, IL-6, IFN- $\gamma$ ) frei und stoßen die Sepsis-Kaskade an.

2. Exotoxine erhöhen die Sensitivität des Organismus ( $\geq 1000$-fach) gegenüber Endotoxinen (z. B. LPS).

3. In-vitro-Untersuchungen weisen zusätzlich auf einen direkt zytotoxischen Effekt von TSST-1 auf Endothelzellen hin und erklären mit die zentrale Bedeutung des Kapillarlecks in der Pathogenese des toxischen Schocksyndroms.

Exotoxine allein können die Pathogenese des toxischen Schocksyndroms nicht erklären. Zusatzfaktoren, vor allem Wirtsfaktoren (z. B. Fehlen bzw. niedrige Titer präexistierender neutralisierender Antikörper gegen die verantwortlichen Exotoxine oder spezifische V $\beta$-Muster des T-ZellRezeptors), spielen eine essenzielle Rolle und bestimmen die Prognose mit. Auch verschiedene humane HLA-KlasseII-Haplotypen schützen vor bzw. prädisponieren zu einem schweren Verlauf einer Infektion mit invasiven Gruppe-AStreptokokken und STSS. 


\section{Klinische Symptome und Verlauf}

Primäre Symptome der Sepsis sind Hyperthermie $\left(>38^{\circ} \mathrm{C}\right)$ oder Hypothermie $\left(<36{ }^{\circ} \mathrm{C}\right)$, Schüttelfrost, Tachykardie und Tachypnoe. Septisches Fieber ist durch Fieberzacken bis $41{ }^{\circ} \mathrm{C}$ charakterisiert, zwischen Körperkerntemperatur (rektal) und peripherer Hauttemperatur ergibt sich häufig eine Differenz von $>3{ }^{\circ} \mathrm{C}$. Im Gesicht imponiert eine ausgeprägte Rötung, die Haut ist initial warm und gut durchblutet. Mit zunehmender Zentralisation des Kreislaufs werden die Extremitäten kühl, die Mikrozirkulation ist gestört. Eine Zyanose zeigt sich häufig zuerst an den Ohren und an der Nasenspitze. Hautveränderungen erlauben gewisse Rückschlüsse auf mögliche Erreger: Petechien oder Purpura bei Meningokokken, Ecthyma gangraenosum bei Pseudomonas aeruginosa. ZNS-Symptome sind Unruhe, Verwirrtheit, Agitiertheit und Angst. Bei fortgeschrittener Sepsis werden die Patienten lethargisch und somnolent. Mit zunehmender Kreislaufinsuffizienz treten Zeichen der Organdysfunktion hinzu. Initiale lokale Symptome können auf einen möglichen Ausgangsfokus hinweisen.

\section{Prognose}

Ein septischer Schock kann sich entweder fulminant innerhalb weniger Stunden entwickeln (z. B. MeningokokkenSepsis oder toxisches Schocksyndrom) oder schleichend verlaufen (z. B. Candida-Sepsis). Die Prognose ist abhängig von Faktoren wie Lebensalter, Grundkrankheit, Art des Erregers, Infektionsherd, Ausmaß der Organbeteiligung und Zeitpunkt des Therapiebeginns. Jenseits des Neugeborenenalters verlaufen $5 \%$ aller Sepsis-Ereignisse tödlich. Je nach Grunderkrankung und Ausmaß einer vorbestehenden Immunsuppression (größtes Risiko: Knochenmarktransplantation) erhöht sich die Rate auf bis zu 40 \%. Ungünstige Prognosefaktoren sind arterielle Hypotension, Koma, Hypothermie, Thrombozytopenie $(<100.000 / \mu \mathrm{l})$, Leukozytopenie $(<5000 /$ $\mu \mathrm{l})$, disseminierte intravasale Gerinnung, eingeschränkte Myokardfunktionen, erniedrigte Antithrombin(AT)-III-Spiegel, erniedrigte Fibrinogen-Spiegel und erhöhte Laktat-, Prokalzitonin-, IL-6-, IL-8-, IL-10-, TNF- $\beta$ - und TREM-1-Spiegel.

Genetische Faktoren (z. B. Polymorphismen in den CD14-, MBL-, TNF- $\alpha-$, IL-1 $\alpha-$, IL-1-Rezeptorantagonist-, IL-6-, IL-8-, IL-10-, Protein-C- und Plasminogen-AktivatorInhibitor-1-Genen) beeinflussen ebenfalls die Prognose der Sepsis.

Die Prognose der Sepsis ist nicht nur durch das Mortalitäts- und Morbiditätsrisiko in der Akutphase bestimmt, sondern auch durch Langzeitkomplikationen (sog. protrahierte Syndrome). Fast jeder 2. Patient mit schwerer Sepsis wird innerhalb des 1. Jahres nach Diagnose einer schweren Sepsis (in der Regel mit Organdysfunktionen) wieder hospitalisiert. Betroffen sind hiervon vor allem Patienten mit onkologischen bzw. immunologischen Grunderkrankungen und hä- matologischen bzw. neurologischen Organdysfunktionen in der Akutphase.

Toxisches Schocksyndrom Beim TSS erkranken typischerweise vorher gesunde Patienten. Der Krankheitsbeginn ist abrupt und der Verlauf ist rasch progressiv. Die Patienten entwickeln Fieber $>38,9^{\circ} \mathrm{C}$, Schüttelfrost und klagen über allgemeine Symptome wie Kopf-, Hals- und Bauchschmerzen, Myalgien, Übelkeit, Erbrechen, Durchfall und Schwindel. Innerhalb von 24-48 Stunden entsteht eine diffuse Erythrodermie, die jedoch bei schwerer arterieller Hypotonie nicht erkennbar sein muss. Der Allgemeinzustand verschlechtert sich rasch, die Patienten entwickeln die klassischen klinischen Zeichen eines Schocks. Das Kapillarleck führt zu generalisierten Ödemen, die Vasodilatation zu einer auffälligen Hyperämie der Schleimhäute der Bindehäute, des Oropharynx und der Vagina. Innerhalb weniger Stunden entsteht das Bild eines fulminanten Multiorganversagens.

Sind die Patienten bei Bewusstsein, fällt eine ausgeprägte muskuläre Druckschmerzhaftigkeit auf. Die Letalität beim TSS liegt auch unter maximaler Therapie bei $2-5 \%$, beim nichtmenstruellen TSS ist sie mit ca. $20 \%$ deutlich höher. Bei Kindern ist die Prognose insgesamt günstiger, 3-5 \% der pädiatrischen Patienten versterben an einem TSS. Überleben die Patienten, so zeigt sich ab dem 5. bis 7. Tag die charakteristische Desquamation an Händen und Füßen, 4-16 Wochen später fallen Haare und Nägel aus. Mögliche Folgeschäden sind neuropsychologische Defizite. Das menstruelle TSS neigt zum Relaps auch ohne erneuten TamponGebrauch.

Etwa $80-85 \%$ der Patienten mit einem STSS haben einen kutanen oder tiefen Weichteilinfektionsherd, seltener einen respiratorischen Infekt als Ursprung des fulminant verlaufenden Organversagens. Unerträgliche Schmerzen und Weichteilschwellung im Bereich der Eintrittspforte sind charakteristisch. Die Letalität ist deutlich höher als beim TSS, sie beträgt 5-30\% im Kindesalter, bzw. (30)-60-(80) \% im Erwachsenenalter und fast $100 \%$ bei parallel bestehender nekrotisierender Fasziitis.

\section{Diagnose}

Für die Diagnose Sepsis ist der klinische Verdacht entscheidend, da sämtliche bisher in der Routine verfügbaren Laborparameter unspezifisch sind. Die Entzündungsparameter Blutsenkungsgeschwindigkeit (BSG), C-reaktives Protein (CRP) und Leukozyten sind erhöht. Eine Differenzierung zeigt vermehrt unreife Vorstufen $(>10 \%)$, das Verhältnis unreifer Vorstufen zu reifen Leukozyten, sog. I:T-Quotient, ist über 0,2 erhöht. Die Leukozyten können toxische Granulationen, Vakuolen und Döhle-Einschlusskörperchen aufweisen. Im Verlauf entwickelt sich häufig eine Leukozytopenie $(<4000 / \mu 1)$, die, wenn sie initial besteht, auf einen schweren Verlauf hinweist. Im Erwachsenenalter ist derzeit 
der beste Marker, um eine bakterielle Sepsis von anderen Ursachen eines SIRS zu unterscheiden, der Nachweis eines erhöhten Prokalzitonin-Spiegels (PCT). Ein PCT-Nachweis von $<0,25-0,5 \mathrm{ng} / \mathrm{ml}$ schließt eine bakterielle Sepsis nahezu aus und kann bei serieller Bestimmung helfen, den Zeitpunkt zur Beendigung einer Antibiotikatherapie festzulegen. Die Rolle des PCTs im Kindesalter ist weniger gut untersucht und kann derzeit nicht abschließend beurteilt werden.

Die Frühphase einer Sepsis, vor allem bei Neu- und Frühgeborenen, wird am zuverlässigsten durch erhöhte ZytokinTiter, z. B. IL-6, IL-8 erfasst. Die Spezifität der Zytokine ist allerdings ähnlich niedrig wie beim CRP oder PCT. Keiner der potenziellen neuen Biomarker, z. B. CD64, pro-Adrenomedullin (proADM) oder soluble triggering receptor expressed on myeloid cell-1 (sTREM-1), hat bisher den Weg in die Routinediagnostik gefunden. Thrombozyten sind sehr häufig vermindert. Bei aktivierter Gerinnung sind die Gerinnungsparameter wie folgt verändert:

- Quick $\downarrow$

- Partielle Thromboplastinzeit (PTT) $\uparrow$

- Fibrinogen $\uparrow$

- Thrombinzeit $\uparrow$

- ATIII $\downarrow$

- Fibrinmonomere $\uparrow$

- Thrombin-Antithrombin-III-Komplex (TAT) $\downarrow$

- Fibrin(ogen)spaltprodukte $\uparrow$

- D-Dimere $\uparrow$

Verlaufskontrolle Folgende klinisch-chemische Parameter können verändert sein und weisen auf Organdysfunktionen hin, sie dienen daher in erster Linie der Verlaufskontrolle:

- Glutamat-Oxalacetat-Transaminase (GOT)

- Glutamat-Pyruvat-Transaminase (GPT)

- $\gamma$-Glutamyl-Transferase $(\gamma$-GT)

- Bilirubin gesamt und direkt

- Kreatinin und Harnstoff

Durch häufige Elektrolytkontrollen muss rechtzeitig eine Hyponatriämie, Hypokaliämie, Hypokalzämie, Hypomagnesiämie, Hypo- oder Hyperglykämie erfasst werden. Die Blutgasanalyse weist anfangs eine kompensierte respiratorische Alkalose bei Tachypnoe auf, im Verlauf entwickelt sich aufgrund der Gewebehypoxie eine metabolische Azidose mit Laktaterhöhung. Die Laktatbestimmung ist wichtig bei der Bestimmung des Schweregrads der Sepsis und stellt einen zentralen Prognosefaktor dar. Bei respiratorischer Verschlechterung besteht eine kombinierte Azidose.

Fokusnachweis In $40 \%$ der Sepsis-Fälle findet sich ein klinischer Fokus, am häufigsten im Respirationstrakt. Bei gegen Pneumokokken, Meningokokken und HiB geimpften Säuglingen und Kleinkindern stehen heute Infektionen der Harnwege im Vordergrund. Bildgebende Verfahren, in erster
Linie Röntgenthorax, Sonografie Abdomen, CT-Abdomen oder MRT-Kopf, dienen zum einem dem Nachweis von Infektionsherden und zum anderen evtl. zur Mitbeurteilung einer Organdysfunktion.

Erregernachweis Zur Erfassung der Ätiologie einer Sepsis gehört der Erregernachweis in entsprechenden Kulturen. Bei klinischem Verdacht auf eine Sepsis sollten mindestens 1 , wenn es der Zustand des Patienten erlaubt, wenigstens 2 Blutkulturen an unterschiedlichen Entnahmestellen (inklusive zentraler Venenkatheter) abgenommen werden. Wichtig ist die Entnahme eines ausreichend großen Blutvolumens (mindestens $1 \mathrm{ml}$, bei größeren Kindern 3-10 ml). Anaerobe Blutkulturen sind nur bei konkretem Verdacht auf Beteiligung von Anaerobiern (z. B. bei abdominellem Infektfokus, Abszessen, Aspirationspneumonie, chronischer Sinusitis, humanen Bisswunden und schwerer Neutropenie oder Mukositis) notwendig. In der Mehrzahl der Fälle bleiben Blutkulturen negativ. Möglicherweise helfen hier zukünftig nichtkulturelle Verfahren, wie die PCR, die in Entwicklung sind. Bei entsprechendem klinischen Infektionsverdacht sollten auch Kulturen von Liquor, Pleura, Aszites, Urin, Trachealsekret (gegebenenfalls auch bronchoalveolärer Lavage) und Abstriche von Wunden und Drainagen entnommen werden.

Toxisches Schocksyndrom Die Diagnose toxisches Schocksyndrom wird aus der Kombination Anamnese, klinischer Befund, Laborkonstellation und Erreger- bzw. Toxinnachweis gestellt (Übersicht „Diagnosekriterien für das Staphylokokken-bedingte toxische Schocksyndrom [TSS; modifiziert nach Wharton et al. 1990]“, Übersicht „Diagnosekriterien für das Streptokokken-bedingte toxische Schocksyndrom [STSS; modifiziert nach der Working Group on Severe Streptococcal Infections 1993]“). Die Anzüchtung von Staphylococcus aureus gelingt beim TSS in $85 \%$ der Fälle aus Vaginalbzw. Zervixsekret oder Wundabstrichen. Blutkulturen sind typischerweise negativ. Speziallabors können die Produktion von TSST-1 oder Staphylokokken-Enterotoxinen der Bakterienisolate nachweisen. Ein solcher Nachweis ist jedoch kein Diagnosekriterium und gelingt durchschnittlich nur in $40 \%$ (bis maximal 60 \%) der Fälle. Allerdings können auch ,normale" Isolate diese Toxine produzieren, sodass im Regelfall eine Bestimmung verzichtbar ist.

Das STSS hingegen fordert zur Diagnosesicherung den Nachweis von Streptokokken aus der Blutkultur (in 60-80\% positiv) oder anderen normalerweise sterilen Untersuchungsmaterialien. Bestimmte Streptokokken-A-Typen werden überzufällig häufig isoliert, z. B. Typ 1, 3, 18. Der Toxinnachweis gelingt aus den meisten Bakterienisolaten. Da sich aber auch bei nichtinvasiven Streptokokken-Stämmen ToxinGene nachweisen lassen, beweist der Toxinnachweis allein keineswegs ein STSS. 


\section{Therapie}

Die frühzeitige Diagnose und Therapieeinleitung einer Sepsis ist der Schlüssel zum Therapieerfolg. Zur kausalen Therapie der Sepsis gehört der frühzeitige und hochdosierte Einsatz von Antibiotika (idealerweise innerhalb der ersten 1-3 Stunden nach Diagnosestellung), nachdem zuvor entsprechende Kulturen gewonnen wurden. Die Auswahl der Substanzen erfolgt initial empirisch und wird später je nach isoliertem Erreger und Antibiogramm modifiziert. Das Antibiotikaregime sollte alle 48-72 Stunden reevaluiert werden und je nach Erregernachweis das Spektrum entsprechend eingeengt werden. Bei der empirischen Auswahl spielen Faktoren wie Alter des Patienten, Immunstatus, Grunderkrankungen, nosokomial erworbene Infektion, Zustand nach Antibiotikatherapie, Kolonisation mit multiresistenten Erregern, lokale Resistenzlage, Gewebegängigkeit und Nebenwirkungsspektrum eine Rolle.

\section{Therapieempfehlungen}

Es gelten folgende Therapieempfehlungen:

- Neugeborene (Kap. „Perinatal und postnatal erworbene Infektionen“) und Säuglinge bis zum 3. Lebensmonat: Ampicillin oder Piperacillin/ $\beta$-Laktamase-Inhibitor plus Aminoglykosid (oder plus Cefotaxim)

- Ab 4. Lebensmonat, ohne Grundkrankheit: Piperacillin/ $\beta$-Laktamase-Inhibitor oder Cephalosporin der Cefotaximgruppe. Aufgrund des assoziierten Risikos einer Selektion von ESBL-bildenden gramnegativen Bakterien sollten Cephalosporine jedoch restriktiv eingesetzt werden

- Nosokomial erworben: Piperacillin/ $\beta$-LaktamaseInhibitor oder Cephalosporin der Ceftazidimgruppe, evtl. plus Aminoglykosid

- Verdacht auf Sepsis mit methicillinresistenten Staphylokokken (MRSA) oder penicillinresistenten Pneumokokken: siehe oben plus Glykopeptid oder Linezolid (bei Verdacht auf MRSA-Pneumonie aufgrund der besseren Gewebegängigkeit bevorzugt einsetzen)

- Infektionsherd im Gastrointestinal- oder Urogenitaltrakt: Piperacillin/ $\beta$-Laktamase-Inhibitor oder $\mathrm{Ce}$ phalosporin der Cefotaxim-/Ceftazidimgruppe, evtl. plus Aminoglykosid. Cephalosporine müssen wegen der intrinsischen Lücke gegen Anaerobier in Kombination mit Metronidazol oder Clindamycin eingesetzt werden

- Immunsuppression oder Neutropenie: Piperacillin/ $\beta$-Laktamase-Inhibitor oder Cephalosporin der

(Fortsetzung)
Ceftazidimgruppe, evtl. mit Aminoglykosid, evtl. mit Glykopeptid (bei erhöhtem Risiko für vergrünende Streptokokken, z. B. nach Hochdosis-Cytarabin-Therapie)

- Katheter-Infektion: Glykopeptid

- Septischer Schock: Meropenem plus Glykopeptid, evtl. plus Aminoglykosid

- Verdacht auf toxisches Schocksyndrom: Flucloxacillin oder Ampicillin/Sulbactam plus Clindamycin (plus intravenöse Immunglobuline [IVIG] bei STSS)

- Verdacht auf Sepsis mit MRGN: Meropenem, evtl. plus Amikacin; Colistin bei Verdacht auf 4MRGN

- Verdacht auf Rickettsiose: Doxycyclin

- Verdacht auf Pilzinfektion (bei Risikofaktoren und fehlendes Ansprechen der Antibiotikatherapie nach 72-96 Stunden): Echinocandin (Caspofungin oder Micafungin) oder liposomales Amphotericin B

- Verdacht auf Herpes-simplex-Virusinfektion: Aciclovir

- Verdacht auf Cytomegalievirus-Infektion: Ganciclovir

Weitere Maßnahmen Neben der antibiotischen Therapie sind weitere Maßnahmen von Bedeutung.

Sanierung des Infektionsfokus Falls möglich sollte eine vorhandene Infektionsquelle beseitigt werden, z. B. durch chirurgische Herdsanierung oder durch Entfernen von Fremdkörpern.

Supportivtherapie Neben der kausalen Therapie spielt die Supportivtherapie eine ganz entscheidende Rolle. Die Behandlung mit kristalloiden und kolloidalen Lösungen, vasoaktiven Medikamenten, Bikarbonat, Sauerstoff und Beatmung wird an anderer Stelle ausführlich besprochen. (Kap.> „Akute Herz-Kreislauf-Insuffizienz und Schock bei Kindern und Jugendlichen").

Für die Sepsis-Behandlung gelten ein paar spezifische Modifikationen:

Eine frühzeitige, zielgerichtete Flüssigkeitstherapie (early goal-directed therapy, EGDT), d. h. Aufrechterhalten eines bestimmten - bislang nur für das Erwachsenenalter definierten - mittleren arteriellen Drucks, zentralen Venendrucks und einer zentralvenösen Sauerstoffsättigung reduziert die Letalität. Bei Kindern werden große Flüssigkeitsboli (unter Umständen bis $>60 \mathrm{ml} / \mathrm{kg} \mathrm{KG/h}$ ) benötigt, um den Kreislauf zu stützen. Eine strikte Blutzuckereinstellung auf Werte von 80-110 mg/dl ist bei Erwachsenen nicht mit einer besseren Überlebenschance verbunden und wird bei Kindern wegen des größeren Risikos für Hypoglykämien nicht empfohlen. 
Eine Insulintherapie wird nach aktueller Datenlage bei Blutzuckerwerten $>180 \mathrm{mg} / \mathrm{dl}$ empfohlen.

Bezüglich der Prophylaxe und Therapie der Verbrauchskoagulopathie gilt zurzeit: Eindeutig indiziert ist die Gabe von Thrombozytenkonzentraten bei Blutungen oder Thrombozytenwerten $<5000 / \mu 1$ sowie die Gabe von Fresh-frozen-Plasma oder Kryopräzipitaten bei Blutungen und pathologischen Quick- oder PTT-Werten. Die Behandlung mit Antithrombin III oder Heparin hat bei Erwachsenen zu keiner Verbesserung der Überlebensraten geführt. Eine generelle Heparinisierung wird nicht empfohlen. Der Hämoglobin-Wert sollte $>10 \mathrm{~g} / \mathrm{dl}$ gehalten werden.

Adjuvante Therapie Eine Vielzahl adjuvanter immunmodulatorischer Substanzen ist bei der Sepsis in Studien erprobt worden, ohne die Prognose der Sepsis entscheidend positiv beeinflusst zu haben. Dieses Therapieversagen hat u. a. zur neuen Prognose-orientierten Sepsis-3-Definition geführt.

Intravenöse Immunglobuline sind nur bei nachgewiesenem IgG-Mangel sowie bei STSS indiziert.

Granulozytentransfusionen sind für Patienten mit schwerer Neutropenie (Kap. \ „Neutrophile Granulozyten - Neutrophilien, Neutropenien und Neutrophilenfunktionsstörungen bei Kindern und Jugendlichen"), die nicht auf eine antimikrobielle Therapie ansprechen, reserviert. Zur Prophylaxe von Infektionen bei Neutropenie kommen Granulozyten-koloniestimulierender Faktor (G-CSF) und Granulozyten-Makrophagen-koloniestimulierender Faktor (GM-CSF) zum Einsatz, bei manifester Sepsis sind sie hingegen ineffektiv.

Hochdosierte Applikation von Kortikosteroiden verbessert die Prognose des septischen Schocks und des frühen ARDS (acute respiratory distress syndrome) nicht und ist daher nicht routinemäßig indiziert. Mögliche Indikationen für eine niedrigdosierte Hydrokortison-Therapie $\left(50 \mathrm{mg} / \mathrm{m}^{2} /\right.$ d) sind der refraktäre septische Schock trotz adäquater Flüssigkeitstherapie und Einsatz von Katecholaminen bei akuter Nebennierenrindeninsuffizienz (z. B. Blutung bei Waterhouse-Friderichsen-Syndrom) sowie die durch Haemophilus influenzae Typ $b$ verursachte Meningitis (zur Verhinderung einer Innenohrschädigung). Die Diagnose einer akuten Nebenniereninsuffizienz sollte durch die Bestimmung eines erniedrigten Serumkortisol-Spiegels $\quad(<150 \quad \mu \mathrm{g} / \mathrm{l} \quad$ bzw. $<90 \mu \mathrm{g} / \mathrm{l}$ nach ACTH-Gabe) gesichert werden.

Die Therapie des Versagens der einzelnen Organe wird in den entsprechenden Kapiteln besprochen.

Spezielle Therapieaspekte beim toxischen Schocksyndrom Die Therapie des toxischen Schocksyndroms gleicht der Therapie der Sepsis. Entscheidend für die Prognose sind das (1) rasche Erkennen der Erkrankung, eine (2) adäquate Supportivtherapie und die (3) rasche Sanierung des Ausgangsherdes. Tampons müssen entfernt, Wunden gespült und drainiert werden. Bei nekrotisierender Fasziitis oder Myositis muss ein großzügiges und frühzeitiges chirurgisches Débridement, evtl. mit Amputation, erfolgen. Zur (4) rationalen Antibiotikatherapie wird bei unbekanntem Erreger eine Kombination aus Flucloxacillin oder Ampicillin/Sulbactam plus Clindamycin empfohlen. Der Einsatz von Clindamycin senkt nachweislich die Letalität beim toxischen Schocksyndrom. Bei MRSA-Verdacht werden statt einem Penicillin- oder Cephalosporin-Präparat Vancomycin oder Teicoplanin als Mittel der 1. Wahl empfohlen. Die obligate Hinzunahme von Clindamycin leitet sich aus folgenden Überlegungen ab:

- Wirksamkeit auch gegen Bakterien in stationärer Wachstumsphase (sog Überwinden des Eagle effects; liegt beim toxischen Schocksyndrom vor),

- Inhibition der Proteinbiosynthese und somit Unterdrückung der Toxinproduktion,

- verbesserte Phagozytose,

- Suppression der TNF- $\alpha$-Sekretion von Monozyten/ Makrophagen,

- längerer postantibiotischer Effekt (im Vergleich zu Penicillinen),

- bessere Gewebegängigkeit (im Vergleich zu Penicillinen).

Nach Erregerisolation kann entsprechend dem Antibiogramm gezielt behandelt werden, Clindamycin (oder alternativ Linezolid) sollte jedoch aufgrund der oben beschriebenen Eigenschaften beibehalten werden. Eine Monotherapie mit Clindamycin ist nicht empfohlen, da es nur bakteriostatisch wirkt.

Die Supportivtherapie entspricht der des septischen Schocks. Der Einsatz von Immunglobulinen (bevorzugt mit $2 \mathrm{~g} / \mathrm{kg} \mathrm{KG}$ als Einzeldosis) wird beim STSS propagiert. Zwei prospektive Beobachtungsstudien aus Schweden bzw. Australien konnten einen Überlebensvorteil bei Einsatz von IVIG beim STSS nachweisen. Die Ergebnisse von retrospektiven Kohortenstudien im Kindesalter sind nicht eindeutig. Eine retrospektive Kohortenstudie aus den USA aus den Jahren 2003-2007 konnte für das Überleben keinen Vorteil in der Immunglobulin-Gruppe zeigen, allerdings waren die Letalitätsraten in der Studienpopulation (mit 4,5\%) ungewöhnlich niedrig. Bei 62 australischen Kindern in den Jahren 2003-2014 war kein einziger Todesfall zu verzeichnen, in $94 \%$ der Fälle hatten die Patienten Clindamycin und/oder IVIG zur adjuvanten Therapie erhalten. Trotz der unklaren Studienlage im Kindesalter wird beim STSS der Einsatz von Immunglobulinen aus theoretischen Überlegungen (Transfer von toxinneutralisierenden Antikörpern) empfohlen.

Ein routinemäßiger Einsatz von Immunglobulinen beim TSS ist umstritten, da die Immunglobulin-Gabe möglicherweise die Bildung protektiver Antikörper unterdrückt und das Risiko eines Rezidives, vor allem beim menstruellen TSS 
Tab. 3 Wesentliche Unterschiede zwischen TSS und STTS

\begin{tabular}{|c|c|c|}
\hline & TSS (toxic shock syndrome) & STSS (streptococcal toxic shock syndrome) \\
\hline \multirow[t]{3}{*}{ Risikofaktoren } & Menses ( $80 \%$ der Betroffenen) & \multirow{3}{*}{$\begin{array}{l}\text { Vorangegangene Haut-, Weichteil-, Atemwegs-, } \\
\text { Virusinfektion (vor allem VZV), Verbrennung, Geburt, } \\
\text { Operation }\end{array}$} \\
\hline & Tamponbenutzung (90\% der Betroffenen) & \\
\hline & $\begin{array}{l}\text { Vorangegangene Haut-, Weichteil-, Atemwegsinfektion, } \\
\text { Verbrennung, Geburt, Abort, Operation }\end{array}$ & \\
\hline Superantigentoxine & $\begin{array}{l}\text { TSST-1 (toxic shock syndrome toxin-1), Staphylokokken- } \\
\text { Enterotoxine A, B, C (SEA, SEB, SEC) }\end{array}$ & $\begin{array}{l}\text { Streptococcal pyrogenic exotoxins (Spe-A, -B, -C), } \\
\text { M-Proteinfragmente }\end{array}$ \\
\hline \multirow[t]{3}{*}{ Letalität } & Menstruelles TSS $2-5 \%$ & Im Erwachsenenalter $30-80 \%$ \\
\hline & Nichtmenstruelles TSS $20 \%$ & Im Kindesalter 5-30\% \\
\hline & TSS im Kindesalter 3-5\% & Bei nekrotisierender Fasziitis fast $100 \%$ \\
\hline Rezidivrisiko & Erhöhtes Rezidivrisiko für menstruelles TSS & Kein erhöhtes Rezidivrisiko \\
\hline Blutkulturen & In der Regel (95\%) negativ für Staphylococcus aureus & In der Regel (60-80\%) positiv für Streptococcus pyogenes \\
\hline $\begin{array}{l}\text { Immunglobulin- } \\
\text { Therapie }\end{array}$ & $\begin{array}{l}\text { Nicht routinemäßig empfohlen (außer bei } \\
\text { therapierefraktären Verläufen) }\end{array}$ & $\begin{array}{l}\text { Empfohlen, vor allem bei persistierender arterieller } \\
\text { Hypotension, nicht drainierbarem Infektionsherd, } \\
\text { persistierender Oligurie, Lungenödem }\end{array}$ \\
\hline Prophylaxe & $\begin{array}{l}\text { Für menstruelles TSS: keine hoch absorbierenden } \\
\text { Tampons, Tampons maximal } 12 \mathrm{~h} \text { belassen, keine } \\
\text { Tampons nach TSS-Erkrankung }\end{array}$ & $\begin{array}{l}\text { Antibiotika-Prophylaxe für Kontaktpersonen von STTS- } \\
\text { Patienten - v. a. mit Risikofaktoren }\end{array}$ \\
\hline
\end{tabular}

VZV Varicella-Zoster-Virus

erhöhen kann. Bei therapierefraktären Verläufen werden Immunglobuline ähnlich zum STSS jedoch ebenfalls propagiert.

\section{Prophylaxe}

Gegen einzelne Sepsis-Erreger gibt es wirksame Impfungen (z. B. Haemophilus influenzae Typ b, Pneumokokken, Meningokokken, Typhus). Treten in der unmittelbaren Umgebung Fälle von Meningokokken oder Haemophilus influenzae auf, erhalten enge Kontaktpersonen eine Antibiotikaprophylaxe (z. B. Rifampicin). Bei sehr kleinen Frühgeborenen $(<1500 \mathrm{~g})$ ist - bei erhöhter lokaler KandidämieInzidenz - der prophylaktische Einsatz von Fluconazol in den ersten 28-45 Lebenstagen sinnvoll. Es reduziert die Kolonisationsrate mit Pilzen und die Rate an invasiven Pilzinfektionen. Ein Überlebensvorteil konnte bisher jedoch nicht gezeigt werden. Der prophylaktische Einsatz von Probiotika bei Frühgeborenen kann das Risiko einer Late-onset-Sepsis reduzieren.

Risikofaktoren für nosokomiale Infektionen sollen minimiert werden, z. B. sollte die Indikation für invasive Interventionen und Katheter streng gestellt werden und ständig überdacht werden. Katheter- oder Drainageeintrittsstellen, sowie Wundflächen müssen regelmäßig inspiziert und bei Infektionsverdacht entsprechend behandelt werden. Die wichtigste Prophylaxe für nosokomiale Sepsis-Fälle ist die strikte Einhaltung von Standardhygienevorschriften.

Toxisches Schocksyndrom Lediglich beim menstruellen TSS sind prophylaktische Maßnahmen nützlich:

- keine Verwendung hoch absorbierender Tampons,

- keine Tampons nach TSS-Krankheit,
- Tampons maximal 12 Stunden belassen.

Spezifische Präventionsmaßnahmen für nichtmenstruelles TSS oder Frauen ohne Tampongebrauch nach menstruellem TSS existieren nicht, abgesehen von einer Aufklärung über Frühsymptome eines TSS und frühzeitigem Aufsuchen ärztlicher Hilfe. Ein Impfstoff gegen TSS mit einer rekombinanten TSST-1-Variante ist in klinischer Entwicklung.

Eine Isolation von Patienten ist nicht notwendig.

Kontaktpersonen von STSS-Patienten haben ein deutlich erhöhtes Risiko (RR 151, 95 \%-KI 79-264) für eine invasive Streptokokken-Infektion, inklusive STSS. Kontrollierte klinische Studien zur Antibiotikaprophylaxe bei Kontaktpersonen liegen nicht vor. Die nationalen Empfehlungen zur Antibiotikaprophylaxe unterscheiden sich. In Kanada wird eine Antibiotikaprophylaxe für alle Kontaktpersonen empfohlen, die USA spricht eine solche nur bei zusätzlichen Risikofaktoren, wie Windpocken, Leukämie oder Verbrennungen (z. B. Penicillin für 7-10 Tage plus Rifampicin für 4 Tage) aus. Unabhängig von der Entscheidung für oder gegen eine Prophylaxe ist eine rasche ärztliche Vorstellung bei Symptomatik einer Streptokokken-Infektion (lokal oder systemisch) dringend geboten (Tab. 3).

\section{Weiterführende Literatur}

\section{Sepsis}

Annane D, Bellisant E, Cavaillon J-M (2005) Septic shock. Lancet 365:63-78

Cornell TT, Wynn J, Shanley TP et al (2010) Mechanisms and regulation of the gene-expression response to sepsis. Pediatrics $125: 1248-1258$ 
Fleischmann C, Thomas-Rueddel DO, Hartmann M et al (2016) Fallzahlen und Sterblichkeitsraten von Sepsis-Patienten im Krankenhaus. Dtsch Ärztebl 113:159-166. https://doi.org/10.3238/arztebl.2016.0159. https://www.ncbi.nlm.nih.gov/pubmed/27010950

Goldstein B, Giroir B, Randolph A et al (2005) International pediatric sepsis consensus conference: definitions for sepsis and organ dysfunction in pediatrics. Pediatr Crit Care Med 6:2-8

S2k-Leitlinie 024-025 „Sepsis bei Kindern jenseits der Neonatalperiode“ (Dezember 2015). http://www.awmf.org/leitlinien/detail/ll 024-025.html

Sáez-Llorens X, McCracken GH (1993) Sepsis syndrome and septic shock in pediatrics: current concepts of terminology, pathophysiology, and management. J Pediatr 123:497-508

Singer M, Deutschmann CS, Seymour CW et al (2016) The third international consensus definitions for sepsis and septic shock (Sepsis-3). JAMA 315:801-810. https://doi.org/10.1001/jama.2016. 0287. https://www.ncbi.nlm.nih.gov/pubmed/26903338

Van der Poll T, van der Veerdonk FL, Sciclina BP et al (2017) The immunopathology of sepsis and potential therapeutic targets. Nat Rev Immunol 17:407-420. https://doi.org/10.1038/nri.2017.36. https://www.ncbi.nlm.nih.gov/pubmed/28436424

\section{Toxisches Schocksyndrom}

Lappin E, Ferguson AJ (2009) Gram-positive toxic shock syndromes. Lancet Infect Dis 9:281-290

McCormick JK, Yarwood JM, Schlievert PM (2001) Toxic shock syndromes and bacterial superantigens: an update. Annu Rev Microbiol 55:77-104

Shah SS, Hall M, Srivastava R et al (2009) Intravenous immunoglobulin in children with streptococcal toxic shock syndrome. Clin Infect Dis 49:1396-1375. https://www.ncbi.nlm.nih.gov/pubmed/19788359

Wharton M, Chorba TL, Vogt RL, Centers for Disease Control (1990) Case definitions for public health surveillance. MMWR Recomm Rep 39(RR-13):1-43. https://www.cdc.gov/mmwr/preview/mmwrht $\mathrm{ml} / 00025629 . \mathrm{htm}$

Wikins AL, Steer AC, Smeesters PR et al (2017) Toxic shock syndromes - the seven Rs of management and treatment. J Infect 74: S147-S152. https://doi.org/10.1016/S0163-4453(17)30206-2. https:// www.ncbi.nlm.nih.gov/pubmed/28646955

Working Group on Severe Streptococcal Infections (1993) Defining the group A streptococcal toxic shock syndrome. Rationale and consensus definition. JAMA 269:390-391. https://www.ncbi.nlm.nih.gov/ pubmed/8418347 